\title{
LA INFRAESTRUCTURA DEL EDIFICIO: Envolventes, instalaciones y sistemas en edificios de oficinas
}

Palabras clave

Chile

Consumo energético

Transparencia

Confort

Transmitancia térmica
Desde que Kahn propusiera la separación

entre espacios servidos y servidores

- transformando a estos últimos en la

infraestructura invisible que permite el

esplendor de los primeros - dicha distinción

se ha mantenido vigente como herramienta

de diseño. Pero cuando los espacios

servidores pasan a contener a los sistemas

del edificio, esta segregación se vuelve

problemática: el edificio ya no sólo depende

de sus propias infraestructuras, sino que

estas también demandan excesiva energía.

- $\mathrm{n}$ los años 6o se introdujo una nueva tipología de edificios en Chile: oficinas con fachadas livianas

(a menudo vidriadas) que descansan en sistemas activos para obtener condiciones de confort. Esto es resultado de una búsqueda global de la arquitectura del siglo $\mathrm{xx}$, que vio un valor en la planta libre, la continuidad espacial o los muros cortina. Sin embargo, a medida que las envolventes se volvieron más ligeras - hasta ser prescindibles estructuralmente - también perdieron la capacidad de aislamiento térmico, acústico y solar que las estructuras de muros sí ofrecían. La búsqueda de un principio estructural idóneo a esta nueva tipología decanta en la integración de sistemas mecanizados, especialmente en edificios de gran altura (Ábalos y 
RENATO D'ALENÇON

Profesor Asistente,

Jefe de Magíster en Arquitectura

Sustentable y Energía, MASE

Escuela de Arquitectura,

Pontificia Universidad Católica

de Chile, Santiago, Chile
Profesor Asociado,

Escuela de Arquitectura, Pontificia

Universidad Católica de Chile,

Santiago, Chile
PEDRO PABLO

DE LA BARRA

Proyecto FONDEF I+D

«Fachada Variable»

Pontificia Universidad Católica

de Chile, Santiago, Chile

Herreros, I992). Así, si la planta libre es soportada por una estructura de pilares, la transparencia y liviandad de la fachada es sostenida por una red de infraestructura de ventilación, aire acondicionado, iluminación artificial, redes de comunicaciones y datos.

\section{El edificio como infraestructura}

Hoy los edificios ya no son objetos singulares, sino productos reproducibles en condiciones urbanas y logísticas semejantes, conformando una infraestructura con rutinas y horarios para organizar la producción y el consumo. Las ciudades y los edificios del mundo entero se construyen de acuerdo a las tecnologías de las infraestructuras (Easterling, 2014).

La definición de infraestructura suele usarse para estructuras, sistemas o redes de gran escala que, si bien son necesarias, se mantienen invisibles o bien aspiran a estar en un segundo plano. Sin embargo, a escala del edificio, la segregación entre sistemas y espacios permite entender que dichos sistemas invisibles son la propia infraestructura del edificio. Así, podemos preguntar: ¿cuáles son y cómo funcionan esas redes de infraestructura en los edificios? ¿Qué implicancia tienen sobre la arquitectura y los cerramientos de los edificios?

En este artículo proponemos analizar la relación entre la arquitectura y la infraestructura del edificio, evaluando qué tan eficiente y eficaz es el calce entre ambos.

Específicamente, nos ocuparemos de la paradoja de los actuales edificios de oficinas en Santiago donde, pese a que el clima templado mediterráneo supone exigencias mínimas, el sobrecalentamiento por radiación y cargas internas, el ruido urbano, la ventilación y renovación de aire, el deslumbramiento de los puestos de trabajo y el consumo energético no han logrado ser resueltos por los sistemas infraestructurales de los edificios.

\section{Los edificios de oficinas en Santiago}

A nivel internacional el consumo energético de los edificios de oficinas está perfectamene cuadrado en el balance energético. La TABLA I describe la situación en Norteamérica, España e Inglaterra, donde los edificios de oficinas representan entre el $2 \%$ y 3,2 \% del consumo global. Especialmente llama la atención el 
perfil del consumo, ya que sobre el 70 \% está asociado

a iluminación y climatización, ambos aspectos

directamente vinculados a la arquitectura de los

edificios. El caso de España es probablemente el que

más se acerque al de Santiago por las condiciones

climáticas, comparables a la zona central de Chile

(Pérez-Lombard, Ortiz y Pout, 2008).

TABLA / CHART 1

Consumo energético en edificios de oficinas, 2003.

Energy consumption for office buildings, 2003.

Fuente / Source Pérez-Lombard et al., 2008

\begin{tabular}{|c|c|c|c|}
\hline Edificios de oficinas & Norteamérica & España & Inglaterra \\
\hline Office buildings & North America & Spain & England \\
\hline $\begin{array}{l}\text { Consumo global respecto al balance energético del país } \\
\text { Global consumption with respect to the country's energy balance }\end{array}$ & $3,2 \%$ & $2,7 \%$ & $2,0 \%$ \\
\hline $\begin{array}{l}\text { Consumo total respecto al sector público y comercial del país } \\
\text { Total consumption with respect to the country's public and commercial sector }\end{array}$ & $18 \%$ & $33 \%$ & $17 \%$ \\
\hline $\begin{array}{l}\text { Consumo de climatización respecto al sector construcción del país } \\
\text { Air conditioning consumption with respect to the country's construction sector }\end{array}$ & $48 \%$ & $52 \%$ & $55 \%$ \\
\hline $\begin{array}{l}\text { Consumo de iluminación respecto al sector construcción del país } \\
\text { Lighting consumption with respect to the country's construction sector }\end{array}$ & $22 \%$ & $33 \%$ & $17 \%$ \\
\hline $\begin{array}{l}\text { Otros consumos de iluminación respecto al sector construcción del país } \\
\text { Other lighting consumption with respect to the country's construction sector }\end{array}$ & $30 \%$ & $15 \%$ & $28 \%$ \\
\hline
\end{tabular}

Gracias a la posibilidad de calcular el comportamiento dinámico de las estructuras sobre I4 pisos, durante los años sesenta comenzó a desarrollarse en Chile la edificación en altura (AUCA, I969a). Pero esta posibilidad estructural implicaba no sólo un desafío para las faenas constructivas, sino también para los sistemas y servicios técnicos en altura. Uno de los primeros ejemplos fueron las torres de Tajamar que, con su edificio principal de 27 pisos de altura, enfrentaron problemas como la implementación de ascensores que duplicaban la altura y velocidad de los previos, o a la necesidad de impulsar agua $\mathrm{y}$ resolver el saneamiento con estrategias hasta entonces inexistentes (AUCA, I969a).

Pero a diferencia de los edificios de viviendas, las torres de oficinas tienen cargas y formas de uso que los convierten en artefactos de una naturaleza distinta, aún en altura. Deben permitir el libre flujo de personas que entran y salen por razones diversas; además, el trabajo concentra a muchas personas en espacios reducidos, tensionando el balance térmico interior $\mathrm{y}$, con ello, las redes de energía. A su vez, la iluminación natural debe cumplir con los estándares requeridos con la mayor uniformidad posible para evitar el uso de iluminación 


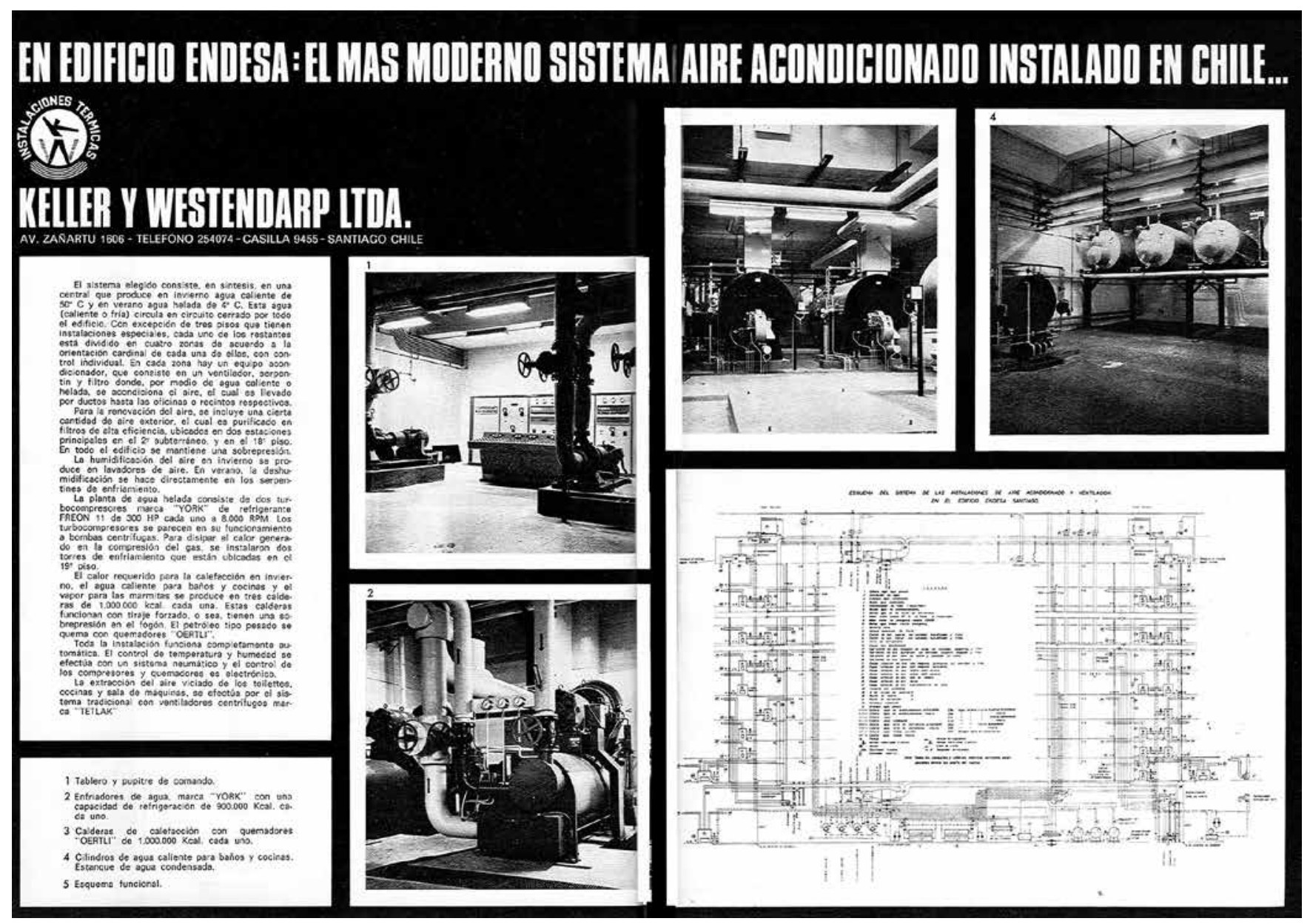

artificial. Por último, se utilizan de forma continua durante el día, lo que obliga a una interacción constante con el clima del lugar donde se emplazan. En Chile, el primero en enfrentar estos problemas fue el edificio Endesa (1965) de los arquitectos Echeverry, Larraguibel y Aguirre (F IG. I). Este edificio fue pionero en varios aspectos: ascensores automatizados (sin ascensorista), climatización centralizada, una central telefónica con I20 líneas y 600 anexos, e iluminación artificial dimensionada para asegurar 500 en todos los puestos de trabajo. En este caso, la estructura de hormigón armado visto se llevó al borde de las losas para dar la mayor libertad posible a las plantas de oficinas, mientras el cerramiento transparente se retiró al plomo interno del pilar para contar con aleros como protección solar. El cerramiento consistió en una fachada liviana de aluminio con ventanas practicables con un 'vidrio polarizado importado' (que hoy debe entenderse como un vidrio tintado). Al ser una experiencia pionera, varios problemas requirieron soluciones especiales por parte de los arquitectos: los ductos de ventilación llevaban el aire de la cocina del casino a las oficinas, el vidrio no proporcionaba el

FIG 1 Anuncio instalación aire acondicionado Keeler y Westendarp en Edificio ENDESA. / Ad for Keeler and Westendarp's air conditioning system in the ENDESA Building. Fuente / Source: AUCA 15 Santiago (agosto, 1969). 
control solar esperado y se debió recubrir las ventanas con una capa adicional de protección, o incluso el sistema de clima no era capaz de reducir la temperatura interior, que llegaba a $26027^{\circ} \mathrm{C}$ (A Av v, I969b). La demanda energética de este edificio también fue un aspecto pionero, pues su consumo en ese momento era comparable al de toda la isla de Chiloé.

Aún así, los edificios de oficinas con fachadas livianas y climatización artificial se transformaron en un estándar en Santiago a partir de los años 9o. Su simpleza tipológica - un núcleo rígido que aloja las circulaciones, un sistema de losas solidarias al núcleo a través de una estructura reticular soportada por pilares en el perímetro y una envolvente adosada a los bordes de las losas daba como resultado una planta libre que permitía una disposición flexible de los espacios de trabajo, en base al modelo de eficacia corporativa consolidado en EE.UU. tras la Segunda Guerra Mundial (Arnold, 2005). Esta configuración, sin embargo, supone una tensión entre el ambiente interior de los edificios y las condiciones climáticas exteriores, donde la radiación solar pone en jaque los anhelados beneficios de la transparencia.

Desempeño de edificios de oficinas en Santiago

En nuestra investigación nos hemos ocupado de caracterizar las redes, las funciones y la envolvente de los edificios de oficinas construidos en Santiago, a través de indicadores que explican su desempeño energético, las cualidades de su forma arquitectónica, las características de su envolvente, la percepción de los usuarios y la calidad de su ambiente interior. Para ello, se definieron cinco clusters o familias de edificios a través del método de clasificación estadística denominado Cluster Analysis (Vásquez, Encinas y D’Alençon, 2015), que permite contar con una visión panorámica del parque construido para estudiarlo sistemáticamente. Las características de los clusters son las siguientes:

- Cluster I: edificios de muro cortina, sin protección solar, de un tamaño superior a los $35.000 \mathrm{~m}^{2} \mathrm{de}$ superficie construida.

- Cluster 2: edificios de fachada mixta, pueden tener o no muro cortina, sin protecciones solares y de superficie en torno a los $10.000 \mathrm{~m}^{2}$.

- Cluster 3: este es un cluster especial con un caso único por su tamaño. Su fachada es un muro cortina sin protección solar.

- Cluster 4: edificios de fachada de muros con vanos y tamaño en torno a los I0.00o $\mathrm{m}^{2}$ de superficie.

- Cluster 5: edificios de muro cortina con algún tipo de protección solar, de un tamaño en torno a los I6.0oo $\mathrm{m}^{2}$ de superficie.

Desde el punto de vista metodológico, hemos realizado auditorías energéticas a casos característicos de cada uno de los cluster, aplicando protocolos ${ }^{1}$ para establecer el perfil del consumo de energía, la calidad de iluminación (natural y artificial), la calidad del ambiente 


\begin{tabular}{|c|c|c|c|c|c|c|c|c|c|c|}
\hline \multirow{2}{*}{$\begin{array}{l}\text { UAE } \\
\left(\mathrm{kWh} / \mathrm{m}^{2} \text { año) }\right.\end{array}$} & \multicolumn{2}{|c|}{ Cluster 1} & \multicolumn{3}{|c|}{ Cluster 2} & \multirow{2}{*}{\begin{tabular}{|c|} 
Cluster 3 \\
Caso 6 \\
Case 6 \\
\end{tabular}} & \multicolumn{2}{|c|}{ Cluster 4} & \multicolumn{2}{|c|}{ Cluster 5} \\
\hline & $\begin{array}{l}\text { Caso } 1 \\
\text { Case } 1\end{array}$ & $\begin{array}{l}\text { Caso } 2 \\
\text { Case } 2\end{array}$ & $\begin{array}{l}\text { Caso } 3 \\
\text { Case } 3\end{array}$ & $\begin{array}{l}\text { Caso } 4 \\
\text { Case } 4\end{array}$ & $\begin{array}{l}\text { Caso } 5 \\
\text { Case } 5\end{array}$ & & $\begin{array}{l}\text { Caso } 7 \\
\text { Case } 7\end{array}$ & $\begin{array}{l}\text { Caso } 8 \\
\text { Case } 8\end{array}$ & $\begin{array}{l}\text { Caso } 9 \\
\text { Case } 9\end{array}$ & $\begin{array}{l}\text { Caso } 10 \\
\text { Case } 10\end{array}$ \\
\hline Caso / Case & 246,83 & 210,01 & 116,30 & 142,02 & 140,45 & 122,01 & 70,46 & 77,42 & 138,98 & 241,47 \\
\hline Cluster & \multicolumn{2}{|c|}{228,42} & \multicolumn{3}{|c|}{132,93} & 122,01 & \multicolumn{2}{|c|}{73,94} & \multicolumn{2}{|c|}{190,22} \\
\hline Total & \multicolumn{10}{|c|}{150,60} \\
\hline
\end{tabular}

térmico interior y la percepción de los usuarios. Todas las mediciones fueron estacionales y se registraron en una base de datos para su posterior análisis.

El protocolo para medir el consumo de energía consideró la medición desagregada de climatización, iluminación y fuerza en algunos pisos de cada caso y la medición del sistema de climatización del edificio en su totalidad. El protocolo de iluminación consideró la medición de iluminancias en los distintos tipos de puesto de trabajo según su relación con las ventanas y su orientación en la planta. El protocolo de calidad del ambiente térmico interior se basó en la zonificación térmica de cada uno de los casos, cruzando información medida instrumentalmente con la percepción de los usuarios.

La TABLA 2 presenta los resultados del protocolo de energía desagregado por caso de estudio, cluster y total. La singular situación del cluster 3 se explica porque la legislación vigente respecto de la tarificación eléctrica (Ministerio de Minería, 20oo; Ministerio de Economía, Fomento y Reconstrucción, 2007) establece que para suministros a usuarios cuya potencia conectada es inferior o igual a $2.000 \mathrm{~kW}$ (considerado un proveedor monopólico) hay precios regulados, pero para suministros a usuarios con potencia conectada superior a $2.000 \mathrm{~kW}$, la ley dispone libertad de precios, suponiendo que ellos tienen capacidad negociadora (CNE, 20I5). Esta diferenciación, propia de un sistema de tarificación que se basa en la asignación eficiente de precios, no considera el uso eficiente de los recursos energéticos, puesto que, en la práctica, introduce un incentivo al mayor consumo para los mayores consumidores.

Como referencia para la evaluación de estos resultados, la TABLA 3 muestra los estándares de consumo de energía para edificios de oficinas en distintos países de la Unión Europea, durante los años en que fueron realizadas las auditorías energéticas.Como vemos, los estándares nacionales son comparables a las referencias de mayor consumo en Europa; sin embargo, el clima es un factor a diferenciar, ya que en la zona central de Chile sólo se podría comparar al de Portugal.

\begin{tabular}{lc}
\hline $\begin{array}{l}\text { País } \\
\text { Country }\end{array}$ & $\begin{array}{l}\text { Estándar }\left(\mathrm{kWh} / \mathrm{m}^{2} \text { año }\right) \\
\text { Standard }\left(\mathrm{kWh} / \mathrm{m}^{2} \text { year }\right)\end{array}$ \\
\hline Austria / Austria & 23 \\
\hline Suiza / Switzerland & 46 \\
\hline República Checa / Czech Republic & 179 \\
\hline Portugal / Portugal & 122 \\
\hline Alemania / Germany & 172 \\
\hline
\end{tabular}




\begin{tabular}{|c|c|c|c|c|c|c|c|c|c|c|c|c|}
\hline \multirow{2}{*}{\multicolumn{2}{|c|}{$\begin{array}{l}\text { Horas de confort } \\
\text { higro-térmico (\%) } \\
\text { Hours of hygrothermal } \\
\text { comfort (\%) }\end{array}$}} & \multicolumn{2}{|c|}{ Cluster 1} & \multicolumn{3}{|c|}{ Cluster 2} & \multirow{2}{*}{\begin{tabular}{|c|} 
Cluster 3 \\
Caso 6 \\
Case 6
\end{tabular}} & \multicolumn{2}{|c|}{ Cluster 4} & \multicolumn{2}{|c|}{ Cluster 5} & \multirow{2}{*}{ Total } \\
\hline & & $\begin{array}{l}\text { Caso } 1 \\
\text { Case 1 }\end{array}$ & Caso 2 & Caso 3 & $\begin{array}{l}\text { Caso } 4 \\
\text { Case } 4\end{array}$ & $\begin{array}{l}\text { Caso } 5 \\
\text { Case } 5\end{array}$ & & $\begin{array}{l}\text { Caso } 7 \\
\text { Case } 7\end{array}$ & Caso 8 & Caso 9 & Caso 10 & \\
\hline \multirow{2}{*}{$\begin{array}{l}\text { Invierno } \\
\text { Winter }\end{array}$} & Caso /Case & $67 \%$ & $95 \%$ & $50 \%$ & $74 \%$ & $48 \%$ & $91 \%$ & $50 \%$ & $52 \%$ & $85 \%$ & $80 \%$ & \multirow{2}{*}{$69 \%$} \\
\hline & Cluster & \multicolumn{2}{|l|}{$81 \%$} & \multicolumn{3}{|l|}{$57 \%$} & $91 \%$ & \multicolumn{2}{|l|}{$51 \%$} & \multicolumn{2}{|l|}{$83 \%$} & \\
\hline \multirow{2}{*}{$\begin{array}{l}\text { Otoño } \\
\text { Autumn }\end{array}$} & Caso /Case & $65 \%$ & $54 \%$ & $14 \%$ & $85 \%$ & $55 \%$ & $93 \%$ & $65 \%$ & $90 \%$ & $78 \%$ & $78 \%$ & $68 \%$ \\
\hline & Cluster & \multicolumn{2}{|l|}{$59 \%$} & \multicolumn{3}{|l|}{$51 \%$} & $93 \%$ & \multicolumn{2}{|l|}{$77 \%$} & \multicolumn{2}{|l|}{$78 \%$} & \\
\hline \multirow{2}{*}{$\begin{array}{l}\text { Primavera } \\
\text { Spring }\end{array}$} & Caso /Case & $73 \%$ & $11 \%$ & $74 \%$ & $78 \%$ & $69 \%$ & $50 \%$ & $16 \%$ & $55 \%$ & $25 \%$ & $30 \%$ & $48 \%$ \\
\hline & Cluster & \multicolumn{2}{|l|}{$42 \%$} & \multicolumn{3}{|l|}{$74 \%$} & $50 \%$ & \multicolumn{2}{|l|}{$35 \%$} & \multicolumn{2}{|l|}{$27 \%$} & \\
\hline \multirow{2}{*}{$\begin{array}{l}\text { Verano } \\
\text { Summer }\end{array}$} & Caso /Case & $79 \%$ & $52 \%$ & $4 \%$ & $58 \%$ & $41 \%$ & $22 \%$ & $66 \%$ & $75 \%$ & $44 \%$ & $53 \%$ & \\
\hline & Cluster & \multicolumn{2}{|l|}{$66 \%$} & \multicolumn{3}{|l|}{$34 \%$} & $22 \%$ & \multicolumn{2}{|l|}{$71 \%$} & \multicolumn{2}{|l|}{$49 \%$} & \\
\hline \multirow{2}{*}{$\begin{array}{l}\text { Anual } \\
\text { Anual }\end{array}$} & Caso /Case & $71 \%$ & $53 \%$ & $35 \%$ & $74 \%$ & $53 \%$ & $64 \%$ & $49 \%$ & $68 \%$ & $58 \%$ & $60 \%$ & \\
\hline & Cluster & \multicolumn{2}{|l|}{$62 \%$} & \multicolumn{3}{|l|}{$54 \%$} & $64 \%$ & \multicolumn{2}{|l|}{$59 \%$} & \multicolumn{2}{|l|}{$59 \%$} & \\
\hline
\end{tabular}

Es importante considerar también que estos estándares de consumo permiten que los edificios logren un alto desempeño de confort higrotérmico y lumínico, una cuestión que es responsabilidad directa de la arquitectura y que, como veremos, no es tal en nuestro caso.

La TABLA 4 muestra los resultados de las mediciones de confort higrotérmico. Las horas de confort térmico corresponden al porcentaje de mediciones puntuales de temperatura y humedad al interior de las plantas dentro del rango de confort específico para cada estación del año según la norma iso $7730\left(20^{\circ} \mathrm{C}\right.$ a $24^{\circ} \mathrm{C}$ en otoño e invierno, $23^{\circ} \mathrm{C}$ a $26{ }^{\circ} \mathrm{C}$ en primavera y verano, humedad relativa entre 20 y $70 \%$ para todo el año). El resultado muestra que la totalidad de los casos tiene un desempeño higrotérmico bajísimo. El caso I, que es el que consumió más energía en el período $\left(246,83 \mathrm{kWh} / \mathrm{m}^{2}\right.$ año), es consecuentemente el que mejor desempeño higrotérmico alcanzó (7I \%), aun cuando sigue siendo bajo respecto a las expectativas de lograr un 10o \% de horas de confort. En general, todos los cluster alcanzan un desempeño en torno al $60 \%$ de horas de confort anual, cuestión que no es coherente con las expectativas de contar con edificios climatizados donde las personas desarrollan actividades de las cuales se espera una cierta productividad.

Finalmente, la TABLA 5 muestra el levantamiento de las horas de confort lumínico, que corresponde al porcentaje de mediciones puntuales de iluminancias al interior de las plantas que se encontraban dentro del rango de confort según la norma iso 8995-I (500 a 2000 lux sobre el plano de trabajo). Los registros se realizaron en los puestos de trabajo representativos para cada orientación y nivel de profundidad dentro de la planta con respecto a la fachada para cada caso de estudio, sobre 200 puntos de medición en la totalidad de la muestra. 


\begin{tabular}{|c|c|c|c|c|c|c|c|c|c|c|c|}
\hline \multirow{2}{*}{\multicolumn{2}{|c|}{$\begin{array}{l}\text { Horas de confort } \\
\text { lumínico (\%) } \\
\text { Hours of light } \\
\text { comfort }(\%)\end{array}$}} & \multicolumn{2}{|c|}{ Cluster 1} & \multicolumn{2}{|c|}{ Cluster 2} & \multirow{2}{*}{\begin{tabular}{|c|} 
Cluster 3 \\
Caso 6 \\
Case 6
\end{tabular}} & \multicolumn{2}{|c|}{ Cluster 4} & \multicolumn{2}{|c|}{ Cluster 5} & \multirow{2}{*}{ Total } \\
\hline & & $\begin{array}{l}\text { Caso } 1 \\
\text { Case } 1\end{array}$ & $\begin{array}{l}\text { Caso } 2 \\
\text { Case } 2\end{array}$ & $\begin{array}{l}\text { Caso } 3 \\
\text { Case } 3\end{array}$ & $\begin{array}{l}\text { Caso } 4 \\
\text { Case } 4\end{array}$ & & $\begin{array}{l}\text { Caso } 7 \\
\text { Case } 7\end{array}$ & $\begin{array}{l}\text { Caso } 8 \\
\text { Case } 8\end{array}$ & $\begin{array}{l}\text { Caso } 9 \\
\text { Case } 9\end{array}$ & $\begin{array}{l}\text { Caso } 10 \\
\text { Case } 10\end{array}$ & \\
\hline \multirow{2}{*}{$\begin{array}{l}\text { Invierno } \\
\text { Winter }\end{array}$} & Caso /Case & $4 \%$ & $8 \%$ & $12 \%$ & $3 \%$ & $15 \%$ & $15 \%$ & $33 \%$ & $30 \%$ & $22 \%$ & \multirow{2}{*}{$15 \%$} \\
\hline & Cluster & \multicolumn{2}{|l|}{$6 \%$} & \multicolumn{2}{|l|}{$7 \%$} & $15 \%$ & \multicolumn{2}{|l|}{$24 \%$} & \multicolumn{2}{|l|}{$26 \%$} & \\
\hline \multirow{2}{*}{$\begin{array}{l}\text { Otoño } \\
\text { Autumn }\end{array}$} & Caso /Case & $5 \%$ & $18 \%$ & $19 \%$ & $2 \%$ & $20 \%$ & $16 \%$ & $12 \%$ & $42 \%$ & $29 \%$ & \multirow{2}{*}{$18 \%$} \\
\hline & Cluster & \multicolumn{2}{|l|}{$11 \%$} & \multicolumn{2}{|l|}{$11 \%$} & $20 \%$ & \multicolumn{2}{|l|}{$14 \%$} & \multicolumn{2}{|l|}{$35 \%$} & \\
\hline \multirow{2}{*}{$\begin{array}{l}\text { Primavera } \\
\text { Spring }\end{array}$} & Caso /Case & $16 \%$ & $11 \%$ & $11 \%$ & $0 \%$ & $20 \%$ & $19 \%$ & $39 \%$ & $36 \%$ & $20 \%$ & $19 \%$ \\
\hline & Cluster & \multicolumn{2}{|l|}{$14 \%$} & \multicolumn{2}{|l|}{$6 \%$} & $20 \%$ & \multicolumn{2}{|l|}{$29 \%$} & \multicolumn{2}{|l|}{$28 \%$} & 1970 \\
\hline \multirow{2}{*}{$\begin{array}{l}\text { Verano } \\
\text { Summer }\end{array}$} & Caso /Case & $15 \%$ & $15 \%$ & $24 \%$ & $0 \%$ & $8 \%$ & $23 \%$ & $20 \%$ & $27 \%$ & $26 \%$ & $17 \%$ \\
\hline & Cluster & \multicolumn{2}{|l|}{$15 \%$} & \multicolumn{2}{|l|}{$12 \%$} & $8 \%$ & \multicolumn{2}{|l|}{$21 \%$} & \multicolumn{2}{|l|}{$26 \%$} & \\
\hline \multirow{2}{*}{$\begin{array}{l}\text { Anual } \\
\text { Anual }\end{array}$} & Caso /Case & $10 \%$ & $13 \%$ & $17 \%$ & $1 \%$ & $16 \%$ & $18 \%$ & $26 \%$ & $34 \%$ & $24 \%$ & $18 \%$ \\
\hline & Cluster & \multicolumn{2}{|l|}{$11 \%$} & \multicolumn{2}{|l|}{$9 \%$} & $16 \%$ & \multicolumn{2}{|l|}{$22 \%$} & \multicolumn{2}{|l|}{$29 \%$} & \\
\hline
\end{tabular}

De la tabla se deduce una baja calidad del ambiente lumínico de los puestos de trabajo, lo que no amerita análisis por el marginal desempeño que alcanzan todos los casos. La disponibilidad de luz (iluminancia) es resultado de un conjunto de factores que combinan la luz natural y sus reflexiones en el espacio, determinados por la profundidad de la planta; así, la iluminación artificial debe funcionar como apoyo en situaciones donde la luz natural no es suficiente. Lo observado en los casos estudiados es que la luz artificial funciona de forma irrestricta durante el día y que el control de la radiación solar normalmente es causa de una luz natural deficiente en los espacios de trabajo. También se debe mencionar que el confort lumínico va más allá de la disponibilidad de luz (iluminancias) y considera la calidad del ambiente lumínico (luminancias); sin embargo, para ninguno de los casos fue posible aplicar protocolos de medición de este aspecto por los bajos niveles de luz disponible.

\section{Conclusiones}

A más de 50 años de la construcción del edificio Endesa, la estrategia de máxima trasparencia de la envolvente soportada por una infraestructura de instalaciones sigue presentando los mismos desafíos que, en Chile, aún no hemos sido capaces de resolver desde el punto de vista del consumo energético ni del confort de los usuarios. La exploración arquitectónica de la transparencia aún no ha dado el paso de problematizar la dimensión de los edificios como componentes de un sistema de infraestructura con el que puede y debe interactuar, en lugar de sólo depender de él.

Los datos presentados dan cuenta de que, en un número significativo de casos estudiados en Santiago, 
la calidad del ambiente interior de los edificios hoy es deficiente a pesar de recibir importantes cantidades de energía. Detrás de esta cuestión es evidente la falta de regulación.

A nivel internacional, las estrategias y regulaciones para abordar estos problemas consideran una doble componente: el sistema de infraestructuras y el desempeño de la envolvente, ambas en referencia a las condiciones requeridas por los usuarios. En Chile, los reglamentos o normas para garantizar el confort ambiental y la eficiencia energética en oficinas siguen siendo casi inexistentes. Las prescripciones de la Ordenanza General de Urbanismo y Construcción (Ministerio de la Vivienda y Urbanismo, I992) se limitan a establecer requisitos de transmitancia térmica para envolventes en viviendas, que no son aplicables a edificios comerciales. Más aún, al considerar edificio y distribución energética como un sistema, las estructuras tarifarias se enfocan en la eficiencia de la designación de precios y no en la eficiencia energética del sistema, en el que los precios puedan funcionar como un incentivo al mejor desempeño térmico o al confort de los usuarios.

Los grandes edificios de oficinas con fachadas transparentes que se construyen hoy en Chile, dependen y forman parte de un sistema de redes de infraestructura (la energética entre otras), y demandan un suministro permanente y en gran cantidad, pero aún no han asumido su rol como parte integrante de él ni su potencial aporte al mejor desempeño y eficiencia del sistema. Sin embargo, si nos enfocamos en los sistemas infraestructurales al interior del edificio, podemos observar que su vida útil relativamente corta $(20$ a 25 años) abre la factibilidad técnica de su sustitución y plantea una oportunidad para reformular los sistemas y la lógica de la operación del edificio. ARQ

\section{Bibliografía / Bibliography}

\footnotetext{
A AVv. «EEdificación en Altura?». AUCA I6 (I969a).

AAVV. «Edificio Endesa.»AUCA I5 (1969b).

ÁBALOS, Iñaki; HER REROS, Juan. Técnica y arquitectura en la ciudad contemporánea, I950-I990. Madrid: Nerea, 1992.

AR NOLD, Thomas. «De la oficina como paisaje al paisaje urbano en la oficina». En Atlas de Edificios de Oficinas, Ed. Rainer Hascher y Simone Jeska. Barcelona: Gustavo Gili, 2005.

Comisión Nacional de Energía. «Tarificación Eléctrica Comisión Nacional de Energía». http://www.cne.cl/en/tarificacion/electrica/. Consultado el 3 de diciembre de 2015

EAS T E R LING, Keller. Extrastatecraft: The Power of Infrastructure Space. London; New York: Verso, 2014.

Ministerio de Economía, Fomento y Reconstrucción. «Reglamento para la fijación de precios de los servicios no consistentes en suministro de energía». Santiago, 2007.
}

Ministerio de la Vivienda y Urbanismo. División Desarrollo Urbano. «Ordenanza General de Urbanismo y Construcciones». Santiago, Chile, I992. http://www.minvu.cl/opensite_20070404I73759.aspx Ministerio de Minería. «Ley General de Servicios Eléctricos». Santiago, 2000.

PÉREZ-LOM BARD, Luis; ORTIZ, José; POUT, Christine. «A Review on Buildings Energy Consumption Information». Energy and Buildings 40 (3, 2008): 394-98.

vÁSQUez, Claudio; encınas, Felipe; D'AlençON, Renato. «Office Buildings in Santiago: What Are We Doing from the Point of View of Energy Consumption?». ARQ 89 (2015), 50-6I. 
I Los protocolos aplicados fueron extraídos de las guías: «Performance measurement protocols for commercial buildings: Best practices guide» $y$ «Performance measurement protocols for commercial buildings», de la American Society of Heating, Refrigerating and Air-conditioning Engineers, ASHRAE; U.s. Green Building Council (USGBC); y The Chartered Institution of Building Services Engineers (CIBSE).

\section{Renato D'Alençon Castrillón}

$<$ dalencon@uc.cl $>$

Arquitecto, Pontificia Universidad Católica de Chile, 1993. Master en Arquitectura, Cornell University, E E.Uu., 2004. Autor del libro Acondicionamientos: Arquitectura y técnica (Santiago de Chile, 2008). Investigador del Centro de Desarrollo Sustentable, CEDEus. Profesor Asistente de la Escuela de Arquitectura de la Pontificia Universidad Católica de Chile, donde es investigador, docente y Jefe de Magíster en Arquitectura Sustentable y Energía, MASE.

\section{Claudio Vásquez Zaldívar}

$<$ cvz@uc.cl>

Arquitecto, Magíster en Arquitectura, Pontificia Universidad Católica de Chile, 1995. Doctor Arquitecto, Universidad Politécnica de Cataluña, España, 2008. Experto en arquitectura sustentable y sistemas de fachadas inteligentes, ha liderado proyectos Fondecyt para el estudio del desempeño energético y ambiental de los edificios de oficinas en Santiago. Fundador y miembro del Grupo de Estudio de Arquitectura y Fachadas Uc. Es Investigador Responsable del proyecto Fondef $\mathrm{I}+\mathrm{D}$ «Fachada Variable: Solución de fachada dinámica en base a patrones de movimiento coordinados para el control solar y lumínico aplicable en Santiago de Chile», además de ser profesor Asociado de la Escuela de Arquitectura uc.

\section{Pedro Pablo de la Barra}

<ppdelaba@uc.cl>

Arquitecto, Magíster en Arquitectura, Pontificia Universidad Católica de Chile, 20r6. Especializado en arquitectura sustentable, con foco en evaluación térmica y lumínica de fachadas. Ha sido ayudante y profesor en distintos cursos del área de edificación y tecnología. Actualmente trabaja como coordinador en el proyecto Fondef $\mathrm{I}+\mathrm{D}$ «Fachada Variable» en la uc. 